\title{
Near-infrared mapping and physical properties of the dwarf-planet Ceres
}

\author{
B. Carry ${ }^{1,2}$, C. Dumas ${ }^{1,3, \star}$, M. Fulchignoni ${ }^{2}$, W. J. Merline ${ }^{4}$, J. Berthier ${ }^{5}$, D. Hestroffer ${ }^{5}$, T. Fusco ${ }^{6}$, and P. Tamblyn ${ }^{4}$ \\ ${ }^{1}$ ESO, Alonso de Córdova 3107, Vitacura, Santiago de Chile, Chile \\ e-mail: bcarry@eso.org \\ 2 LESIA, Observatoire de Paris-Meudon, 5 place Jules Janssen, 92190 Meudon Cedex, France \\ 3 NASA/JPL, MS 183-501, 4800 Oak Grove Drive, Pasadena, CA 91109-8099, USA \\ 4 SwRI, 1050 Walnut St. \# 300, Boulder, CO 80302, USA \\ 5 IMCCE, Observatoire de Paris, CNRS, 77 Av. Denfert Rochereau, 75014 Paris, France \\ ${ }^{6}$ ONERA, BP 72, 923222 Châtillon Cedex, France
}

Received 26 June 2007 / Accepted 6 November 2007

ABSTRACT

\begin{abstract}
Aims. We study the physical characteristics (shape, dimensions, spin axis direction, albedo maps, mineralogy) of the dwarf-planet Ceres based on high angular-resolution near-infrared observations.

Methods. We analyze adaptive optics $J / H / K$ imaging observations of Ceres performed at Keck II Observatory in September 2002 with an equivalent spatial resolution of $\sim 50 \mathrm{~km}$. The spectral behavior of the main geological features present on Ceres is compared with laboratory samples.

Results. Ceres' shape can be described by an oblate spheroid ( $a=b=479.7 \pm 2.3 \mathrm{~km}, c=444.4 \pm 2.1 \mathrm{~km})$ with EQJ2000.0 spin vector coordinates $\alpha_{0}=288^{\circ} \pm 5^{\circ}$ and $\delta_{0}=+66^{\circ} \pm 5^{\circ}$. Ceres sidereal period is measured to be $9.07410_{-0.00014}^{+0.000} \mathrm{~h}$. We image surface features with diameters in the $50-180 \mathrm{~km}$ range and an albedo contrast of $\sim 6 \%$ with respect to the average Ceres albedo. The spectral behavior of the brightest regions on Ceres is consistent with phyllosilicates and carbonate compounds. Darker isolated regions could be related to the presence of frost.
\end{abstract}

Key words. minor planets, asteroids - infrared: solar system - techniques: high angular resolution - methods: observational

\section{Introduction}

Ceres is by far the largest body among the population of mainbelt asteroids. Curiously, although it was discovered more than $200 \mathrm{yr}$ ago (Piazzi 1802), significant progress to understand its surface and interior properties has only been made over the last two decades. In particular we have seen a renewal of interest towards Ceres triggered by (i) the availability of sensitive spectroimaging instrumentation on medium-to-large size telescope, enabling a detailed study of Ceres surface and physical properties (e.g. Mitchell et al. 1996; Drummond et al. 1998; Dotto et al. 2000, and others); and (ii) more recently the selection of the NASA DAWN Discovery mission, which will visit Vesta and Ceres in 2011 and 2015 respectively (Russell et al. 2003). But the main scientific interest drawn by Ceres is that it provides an excellent laboratory to understand how planetoids accreted early in the history of our solar system, and the role of volatiles in planetary formation and evolution. Unlike Vesta, which is dry and shows evidence of the melting phase and planetary differentiation (Keil 2002) seen in larger terrestrial planets like Earth, the "dwarf planet" Ceres (as it should now be named following the IAU guidelines) shows strong signs of water alteration on its surface (Jones et al. 1990). In this respect, Ceres displays stronger similarities with the icy outer satellites of Jupiter than with the dry asteroids that populate the inner region of the Main Belt. A possible scenario is that Ceres formed in a "wet" environment, from the accretion of both rocky planetesimals originally present

\footnotetext{
* Affiliation at the time the observations were obtained.
}

at this heliocentric distance in the early planetary nebula, and icy planetesimals that migrated inward from the outer regions and whose ices had been preserved (Mousis \& Alibert 2005). As a consequence of this "wet" history, the study of Ceres is of paramount importance to understand the process of planetary accretion and formation of the low-albedo primitive asteroids that populate the outer part of the Main Belt.

With a typical angular diameter of $0.6^{\prime \prime}$ at opposition, Ceres can be spatially resolved from the ground using adaptive optics instruments available on medium-to-large telescopes. We carried out a program of multi-band imaging of Ceres from Keck Observatory at high angular-resolution, with the goals of precisely deriving its shape, dimensions, direction of spin axis, and distributions of albedo and color across its surface.

\section{Observations and data reduction}

\subsection{Observations}

Our Ceres observations were made with the Keck II telescope on Mauna Kea, Hawaii, on 2002 September 22 and 28 UT, one week before the opposition of Ceres. These observations were obtained under optimal atmospheric conditions with a subarcsec seeing at an airmass lower than 1.65 (with half of the data taken with an airmass lower than 1.2). The 2002 opposition occured near Ceres aphelia (Ceres was at a geocentric and heliocentric distance of $1.98 \mathrm{AU}$ and $2.94 \mathrm{AU}$ respectively), its apparent angular diameter was $\sim 0.66^{\prime \prime}$. The phase angle was of $7^{\circ}$ and $5.5^{\circ}$ for the two nights, leading to an illuminated fraction of the surface of $99.6 \%$ and $99.7 \%$ respectively. 
Ceres was imaged using NIRC2, the second-generation nearinfrared camera $(1024 \times 1024 \mathrm{InSb}$ Aladdin-3) and the adaptive optics (AO) system installed at the Nasmyth focus of the Keck II telescope (van Dam et al. 2004). The images of Ceres were acquired at 3 near-infrared wavebands $J[1.166-1.330 \mu \mathrm{m}]$, $H[1.485-1.781 \mu \mathrm{m}]$, and $K[1.948-2.299 \mu \mathrm{m}]$, with an image scale of $9.942 \pm 0.050$ milliarcsec per pixel. Within the Ceres observation sequence we interspersed observations of reference stars, at similar airmass and through the same set of filters, to evaluate the system Point Spread Function (PSF). This calibration was required to perform a posteriori image restoration as described in the next section. No offset to sky was done but the telescope position was dithered between each exposure in order to record simultaneous sky and object frames, while the target (science or calibration) was positioned at three different locations on the detector, separated by nearly $5^{\prime \prime}$ from each other.

\subsection{Data reduction}

We first reduced the data using the standard procedure for nearinfrared images. A bad pixel mask was made by combining the hot and dead pixels found from the dark and flatfield frames. The bad pixels in our calibration and science images were then corrected by replacing their values with the median of the neighboring pixels $(7 \times 7$ pixel box $)$. Our sky frames were obtained from the median of each series of dithered science image, and then subtracted from the corresponding science images to remove the sky and instrumental background. By doing so, the dark current was also removed. Finally, each image was divided by a normalized flatfield to correct the pixel-to-pixel sensitivity differences of the detector.

After these first basic reduction steps, we applied image deconvolution techniques to our set of Ceres data using the MISTRAL algorithm (Fusco 2000; Mugnier et al. 2004). The use of such an algorithm permits to restore the optimal spatial resolution of each image and is particularly well adapted to deconvolve objects with sharp edges, such as asteroids. Image restoration techniques are known to be constrained by the limitations to measure the precise instrumental + atmosphere responses at the exact same time the science observations are made. MISTRAL is a myopic deconvolution method, which estimates both the most probable object, and the PSF, from analysis of science and reference star images. The time needed for the algorithm to converge is largely dependent on the image size. Due to our large number of images to process, we decreased the deconvolution computation time by resizing all our images to a smaller $(128 \times 128$ pixels, but still over $2 \times$ the diameter of Ceres) window centered on the object (Ceres or PSF). Comparison tests showed that no deconvolution artifacts were introduced by the use of smaller images. The deconvolved images of Ceres were then compared to identify the few frames ( 9 in total, that is less than $2.5 \%$ of the full set of data) whose outputs were not satisfactory and discard them from our set of data. In the end we obtained 360 images of Ceres with a spatial resolution (Table 1) equivalent to the diffraction limit of a $10 \mathrm{~m}$ telescope (given by the angular sampling $\Theta=\lambda / D$, with $\lambda$ the wavelength and $D$ the telescope diameter). A subset of the restored images is presented in Fig. 1.

\section{Ceres global physical properties}

\subsection{Spin vector coordinates}

Measuring the Spin Vector Coordinates (SVC) of Ceres has always been a difficult task. The small amplitude of its
Table 1. Central wavelength $\left(\lambda_{\mathrm{c}}\right)$ and bandpass width $(\Delta \lambda)$ for each filter. The equivalent size (in $\mathrm{km}$ ) of the theoretical resolution element $(\Theta)$ on Ceres and the number of resolution elements across the diameter and over the apparent disk of Ceres are also reported. Ceres covers more than 3200 pixels (projected major- and minor-radius of $\sim 33$ and $\sim 31$ pixels respectively) on the NIRC2 detector.

\begin{tabular}{cccccc}
\hline \hline Filter & $\begin{array}{c}\lambda_{\mathrm{c}} \\
(\mu \mathrm{m})\end{array}$ & $\begin{array}{c}\Delta \lambda \\
(\mu \mathrm{m})\end{array}$ & $\begin{array}{c}\Theta \\
(\mathrm{km})\end{array}$ & $\begin{array}{c}\Theta \text { Across } \\
\text { diameter }\end{array}$ & $\begin{array}{c}\Theta \text { Over } \\
\text { surface }\end{array}$ \\
\hline$J$ & 1.248 & 0.163 & 37.2 & $\sim 26$ & $\sim 666$ \\
$H$ & 1.633 & 0.296 & 47.9 & $\sim 20$ & $\sim 400$ \\
$K$ & 2.124 & 0.351 & 64.6 & $\sim 15$ & $\sim 228$ \\
\hline
\end{tabular}

lightcurve ( $\sim 0.04 \mathrm{mag}$ ) prevented its determination using classical lightcurve inversion techniques. Until recently, the solutions that were reported for the pole coordinates of Ceres were widely dispersed (spanning a $90^{\circ}$ range in the plane of the sky during the 1995 opposition as reported by Parker et al. (2002), based on the compilation of Johnson et al. (1983), Millis et al. (1987), Saint-Pé et al. (1993) and Drummond et al. (1998) pole solutions). This situation changed dramatically with the use of highcontrast direct imaging techniques capable of resolving the disk of Ceres and image fine details on its surface, such as AO observations (e.g. Dumas et al. 2003, and this paper) and HST observations (e.g. Parker et al. 2002; Thomas et al. 2005). The high-quality data obtained at Keck allow us to obtain an independent measurement of the SVC of Ceres, which is compared to the HST results (Thomas et al. 2005). Precise knowledge of the SVC is mandatory to re-project the individual images into Ceres planetocentric referential and establish albedo maps of its surface (see Sect. 4.2).

We performed a control-point method, which consists of following the apparent motion of a surface feature while the asteroid rotates. The path of a rotating feature on the surface of Ceres describes an ellipse if the asteroid is seen under a SubEarth Point latitude $\left(\mathrm{SEP}_{\varphi}\right)$ different from $0^{\circ}$, or $\pm 90^{\circ}$ (where it describes a line, or a circle respectively). We marked the positions of two main features A and B (Fig. 1) while they rotated respectively over a $93.9^{\circ}$ and $87.7^{\circ}$ range around Ceres' spin axis (100 and 87 images respectively). All these measurements are shown together on a 3-D representation of Ceres in Fig. 2. The shape of the projected track being determined by the direction of the rotational axis, we were able to measure the north pole angle $\left(p_{n}\right)$ and the $\operatorname{SEP}_{\varphi}$ at the time of the observations.

In order to compare our solution with other results, we followed the IAU recommendations and calculated the SVC in the J2000.0 equatorial frame: $\alpha_{0}=288^{\circ} \pm 5^{\circ}$ and $\delta_{0}=+66^{\circ} \pm 5^{\circ}$. This result is in agreement with the latest result from HST (Thomas et al. 2005) $\left(\alpha_{0}=291^{\circ} \pm 5^{\circ}\right.$ and $\left.\delta_{0}=+59^{\circ} \pm 5^{\circ}\right)$.

To directly derive the obliquity of a planetary body, it is also convenient to express the SVC in an ecliptic reference frame. Our measurements give $\lambda_{0}=7^{\circ}$ and $\beta_{0}=+83^{\circ}$ (with $5^{\circ}$ uncertainty), leading to an obliquity for Ceres of about $4^{\circ}$. Such a small obliquity was expected from analysis of lightcurve data (Tedesco et al. 1983) and thermal properties of Ceres regolith (Spencer 1990). Indeed, a larger obliquity would imply stronger variations in the amplitude of Ceres' lightcurves, which have never been reported. As a result, Ceres can only be observed over a small range of Sub-Earth Point latitude $\left(\operatorname{SEP}_{\varphi}\right)\left(-11^{\circ}\right.$, $\left.+10^{\circ}\right)($ computed until 2015), we must wait for the arrival of the DAWN spacecraft to get a direct view of its polar regions. 


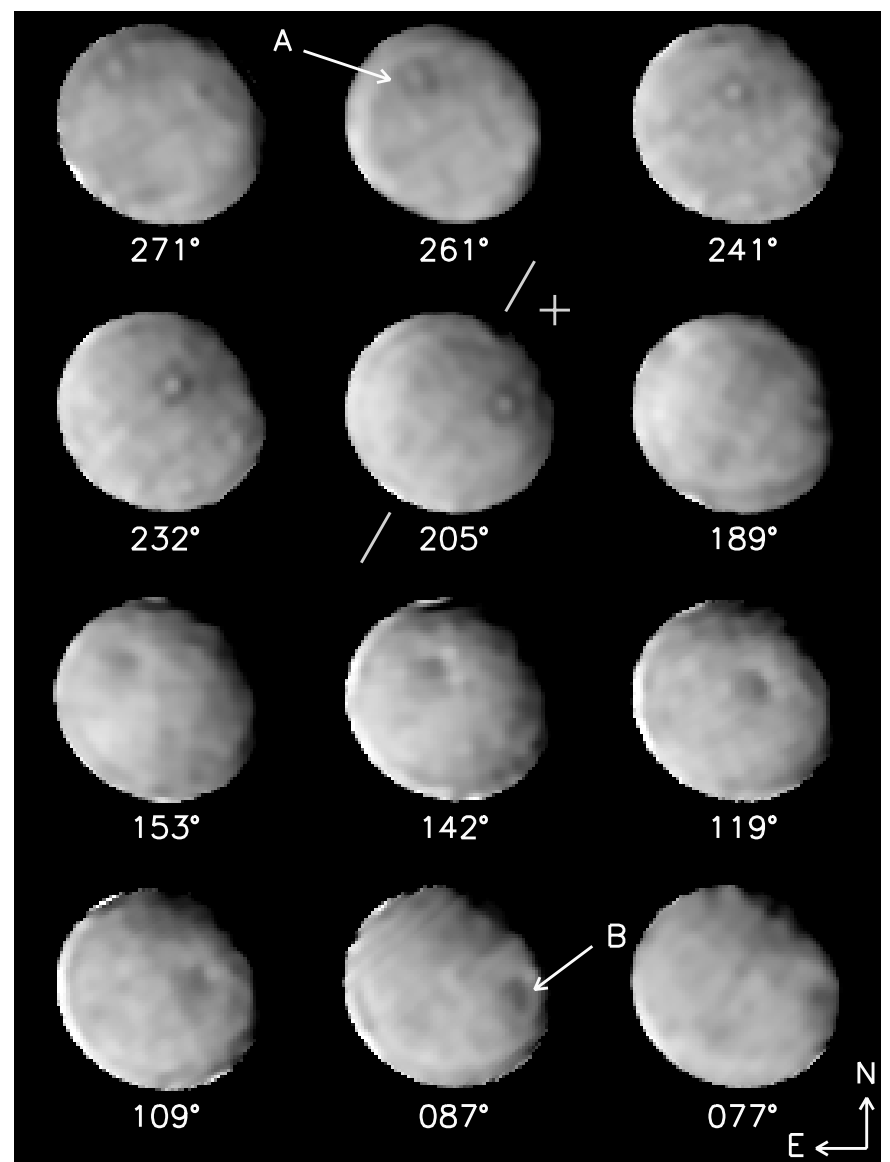

Fig. 1. Selected views of Ceres in $K$-band during $\sim 200^{\circ}$ of its rotation. The image is oriented with North up and East left. The values of the Sub-Earth Point longitude $\left(\mathrm{SEP}_{\lambda}\right)$ measured positively from 0 to 360 degrees in a right-hand system (following the IAU recommandation Seidelmann et al. 2005) are indicated below their corresponding images. Our meridian origin is chosen to be the same than Li et al. (2006a). The Ceres spin axis and positive pole are also indicated. The image stretch was chosen to enhance the surface features visible on Ceres at the detriment of the terminator. The two main surface features present on Ceres are indicated with the arrows A and B (see Sect. 3.1) and can be followed during part of their rotation. The brighter spots visible near the limb of Ceres in some of the images are artifacts from the deconvolution (see Sect. 4.2).

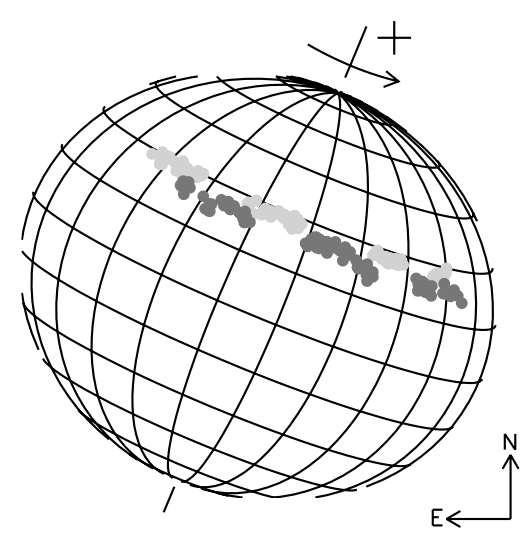

Fig. 2. Apparent motion of the two main features A (bright) and B (dark) over $\sim 90^{\circ}$ of Ceres rotation during the night of the 2002 September $22 \mathrm{UT}\left(\operatorname{SEP}_{\varphi} \simeq+8^{\circ}, p_{n} \simeq 338^{\circ}\right)$. We used the rotational track of these two features to determine the direction of the rotation axis (as described in text).
Table 2. Sub-Earth Point longitude $\left(\mathrm{SEP}_{\lambda}\right)$ for the three epochs $\left(t_{1}\right.$ and $t_{2}$ from Keck, $t_{3}$ from HST). Using $t_{1}$ as reference, Ceres sidereal phasing with $t_{2}$ and $t_{3}$ can be obtained by applying a $+1.3^{\circ}$ and a $-98.7^{\circ}$ correction taking into account the apparent geometry of the Earth with respect to Ceres.

\begin{tabular}{ccc}
\hline \hline Epoch & $\begin{array}{c}\text { Date } \\
\text { (Julian Day) }\end{array}$ & $\begin{array}{c}\mathrm{SEP}_{\lambda} \\
\left({ }^{\circ}\right)\end{array}$ \\
\hline$t_{1}$ & 2452539.89402 & $234.6 \pm 2$ \\
$t_{2}$ & 2452545.91546 & $261.4 \pm 2$ \\
$t_{3}$ & 2453002.24128 & $22.6 \pm 5$ \\
\hline
\end{tabular}

\subsection{Rotational period}

We establish a precise and independent measurement of the rotation period of Ceres using images from our two Keck epochs (2002 September 22 and 28 UT), plus one additional processed Hubble ACS/HRC image (j8p502amq_iof.fit) from the HST program GO 9748 retrieved from the Small Body Nodes archive (Li et al. 2006b) for the epoch 2003 December 28 UT (Table 2) (Thomas et al. 2005; Li et al. 2006a; Parker et al. 2006). Two main albedo marks visible in all images were used to obtain precise information on Ceres rotational phase at these three epochs (see Table 2). In addition, the period between the Keck and HST observations was sufficiently large to accurately measure the error accumulation on Ceres' period over more than 1000 rotations.

We then used the Eproc ephemeris generator (Berthier 1998) to predict the longitude of the Sub-Earth Point and determine the value of the Ceres sidereal period that minimizes the difference $\triangle \mathrm{SEP}_{\lambda}$ between the observed and the computed $\mathrm{SEP}_{\lambda}$. We adopted the pole solution derived in this work as well as the priorly determined Ceres sidereal period of $P_{\mathrm{s}}=9.075 \pm 10^{-3} \mathrm{~h}$ (Tedesco et al. 1983) as a best estimate. Table 3 shows the sidereal periods that are in agreement with the Sub-Earth Point longitude $\left(\operatorname{SEP}_{\lambda}\right)$ at the epochs of the observations.

The value of Ceres sidereal period best matching our observations was found to be $P_{\mathrm{s}}=9.07410_{-0.00014}^{+0.00010} \mathrm{~h}$ with a $\sim 10^{-4} \mathrm{~h}$ resolution. The small $\sim 0.5 \mathrm{~s}$ uncertainty, which come mainly from the error on the mesured longitudes (see Table 2), makes possible to predict Ceres' Sub-Earth Point longitude, $\mathrm{SEP}_{\lambda}$, with an error of only $40^{\circ}$ in rotational phase over the next decade. By comparison, the recent study by Chamberlain et al. (2007) compiled $50 \mathrm{yr}$ of lightcurve measurements to derive a period of $P_{\mathrm{s}}=9.074170 \pm 2 \times 10^{-6} \mathrm{~h}$.

\subsection{Dimensions}

Precise measurements of the shape of Ceres allow us to remotely investigate its internal structure and test whether it is a differentiated body (Thomas et al. 2005). We thus performed a Laplacian of Gaussian (LoG) wavelet analysis to 192 images of Ceres to extract its limb contours. Because it is very sensitive to variations of gradient, the LoG permits to precisely detect the inflection points in the flux distribution of our individual deconvolved images of Ceres. We found Ceres to be rotationally symmetric as first reported from a preliminary analysis of the Keck-AO data by Dumas et al. (2003), and later confirmed by Thomas et al. (2005). Analysis of our complete set of contours did not return any deviation from our ellipsoidal model larger than $\sim 18 \mathrm{~km}$ (see Fig. 3). From this model, and our knowledge of the direction of its spin axis, we were able to determine the minor- and major- projected radius of Ceres and correct them from their 
Table 3. Sidereal periods of Ceres which are in agreement with our observations, based on $\operatorname{SEP}_{\lambda}\left(t_{1}\right)=234.6^{\circ}$. The predicted Sub-Earth Point longitude $\left(\mathrm{SEP}_{\lambda}\right)$ are computed at epochs $\mathrm{t}_{2}$ and $\mathrm{t}_{3}$ and are compared with the measurements (difference $\triangle \mathrm{SEP}_{\lambda}$ ). We only kept sidereal periods whose predicted $\operatorname{SEP}_{\lambda}(\mathrm{t})$ were inside measurement uncertainties.

\begin{tabular}{ccccc}
\hline \hline $\begin{array}{c}\text { Sidereal period } \\
(\mathrm{h})\end{array}$ & $\begin{array}{c}\mathrm{SEP}_{\lambda}\left(\mathrm{t}_{2}\right) \\
\left({ }^{\circ}\right)\end{array}$ & $\begin{array}{c}\Delta \mathrm{SEP}_{\lambda} \\
\left({ }^{\circ}\right)\end{array}$ & $\begin{array}{c}\mathrm{SEP}_{\lambda}\left(\mathrm{t}_{3}\right) \\
\left({ }^{\circ}\right)\end{array}$ & $\begin{array}{c}\Delta \mathrm{SEP}_{\lambda} \\
\left({ }^{\circ}\right)\end{array}$ \\
\hline 9.066588 & 255.1 & 6.3 & 17.9 & 4.7 \\
9.066685 & 255.1 & 6.3 & 22.6 & 0.0 \\
9.066780 & 255.1 & 6.3 & 27.2 & -4.6 \\
\hline 9.074000 & 259.8 & 1.6 & 17.8 & 4.8 \\
9.074090 & 259.8 & 1.6 & 22.1 & 0.5 \\
9.074100 & 259.8 & 1.6 & 22.6 & 0.0 \\
9.074110 & 259.8 & 1.6 & 23.1 & 0.5 \\
9.074200 & 259.9 & 1.6 & 27.5 & -4.9 \\
\hline 9.081526 & 264.4 & -3.0 & 17.9 & 4.7 \\
9.081526 & 264.5 & -3.1 & 22.6 & 0.0 \\
9.081526 & 264.6 & -3.2 & 27.2 & -4.6 \\
\hline
\end{tabular}

Table 4. Ceres sidereal period $\left(P_{\mathrm{s}}\right)$, pole direction $\left(\alpha_{0}, \delta_{0}\right)$ and initial rotational phase angle $\left(W_{0}\right)$ at epoch of reference in the $\mathrm{J} 2000.0$ equatorial frame.

\begin{tabular}{ccccc}
\hline \hline $\begin{array}{c}\alpha_{0} \\
\left(^{\circ}\right)\end{array}$ & $\delta_{0}$ & $W_{0}$ & $P_{\mathrm{s}}$ & $\begin{array}{c}\text { Epoch } \\
\left({ }^{\circ}\right)\end{array}$ \\
\hline $288 \pm 5$ & $+66 \pm 5$ & $-46 \pm 2$ & $9.0741 \pm 10^{-4}$ & 2452539.89402 \\
\hline
\end{tabular}
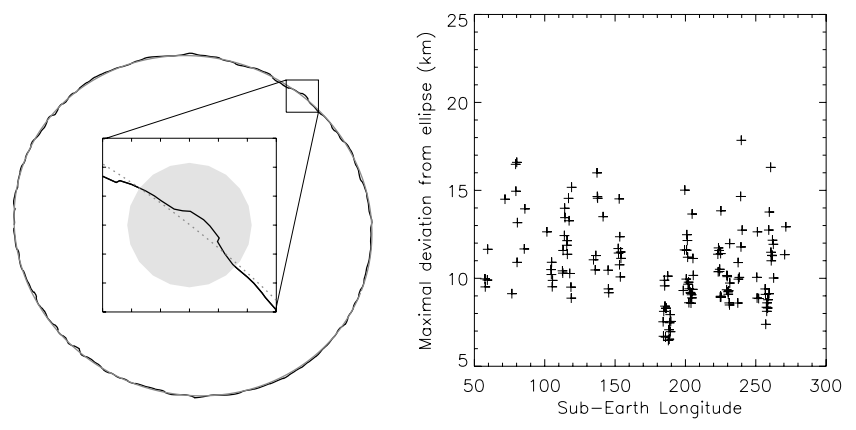

Fig. 3. Left figure: extracted limb contour (black) from a $J$-band image of Ceres with its ellipsoidal fit overplotted for comparison (dotted gray). This example shows that deviations from the ellipsoidal model are much smaller than the resolution element (gray disk inside the box) obtained with Keck-AO. Right figure: compilation of our deviation measurements as function of the Sub-Earth Point longitude $\left(\mathrm{SEP}_{\lambda}\right)$. No deviations larger than $\sim 18 \mathrm{~km}$ (half a resolution element at $J$-band) are detected.

aspect and phase angles. We found that Ceres is well described by an oblate spheroid whose semi-axes are $a=b=$ $479.7 \pm 2.3 \mathrm{~km}$ and $c=444.4 \pm 2.1 \mathrm{~km}(1-\sigma$ dispersion for the fitted axis). These values are different from HST's by $\sim 10 \mathrm{~km}$ (relative difference of two percent). In comparison, the agreement between our semi-major axis and the determination made from stellar occultation by Millis et al. (1987) is remarkable: $479.6 \pm 2.4 \mathrm{~km}$ (occultation) vs. $479.7 \pm 2.3 \mathrm{~km}$ (Keck), while our minor-axis value differ from theirs by $\sim 6-$ $9 \mathrm{~km}$ (Table 5). Whereas Millis et al. (1987) assumed a zeroobliquity at the time of the occultation, our SVC solution gives a $\mathrm{SEP}_{\varphi}$ of $+3.3^{\circ}$. The corrected polar radius from stellar occultation is thus $453.3 \pm 4.5 \mathrm{~km}$, which is still different from our measurement by nearly $10 \mathrm{~km}$. It is important to note that during the occultation of 1987, the cords along Ceres orbit were aligned in an East-West direction, whereas the north
Table 5. Semi-major $(a)$ and -minor $(c)$ axes, and equivalent radius $(R=\sqrt[3]{a a c})$ for Ceres derived in this paper (Keck), Thomas et al. (2005) (HST), and Millis et al. (1987) (Occultation). The pixel size of the Keck and HST images are also given for comparison.

\begin{tabular}{ccccc}
\hline \hline Quantity & \multicolumn{2}{c}{ Imagery } & \multicolumn{2}{c}{ Occultation } \\
$(\mathrm{km})$ & Keck & HST & Sol. 1 & Sol. 2 \\
\hline$a$ & $479.7 \pm 2.3$ & $487.3 \pm 1.8$ & $479.6 \pm 2.4$ & $481.6 \pm 2.4$ \\
$c$ & $444.4 \pm 2.1$ & $454.7 \pm 1.6$ & $453.4 \pm 4.5$ & $450.1 \pm 2.0$ \\
$R$ & $467.6 \pm 2.2$ & $476.2 \pm 1.7$ & $470.7 \pm 3.1$ & $470.8 \pm 2.3$ \\
pixel & $14.3 \pm 0.7$ & $33.9 \times 29.6$ & $\ldots$ & $\ldots$ \\
\hline
\end{tabular}

pole position angle was $p_{n} \sim 339^{\circ}$. This implies that the estimation of the small radius of Ceres was more loosely constrained than its semi-major axis. In addition, Table 5 provides a comparison between the pixel size of the HST and Keck images, and shows that the coarser sampling of the disk of Ceres as seen by HST could explain the differences in our respective determinations of its size. Our mean radius for Ceres is estimated to be $R=467.6 \pm 2.2 \mathrm{~km}$, which is also closer to the determination made from stellar occultation than HST's.

Adopting a mass for Ceres of $M=9.43 \pm 0.07 \times 10^{20} \mathrm{~kg}(\mathrm{av}-$ erage of most recent measurements, Viateau \& Rapaport 1998; Michalak 2000; Kovačević \& Kuzmanoski 2007), we find a mean density $\rho=2206 \pm 43 \mathrm{~kg} \mathrm{~m}^{-3}$. This value is relatively high for a hydrated G-type asteroid like Ceres, but can be explained by a low porosity (see Britt et al. 2002), and is similar to the density of the icy outer Jovian satellites Ganymede and Callisto. One can assume Ceres to be in hydrostatic equilibrium and inverse the relation between $a, c$ and $J_{2}$ (given by McCord \& Sotin 2005) as following

$J_{2}=\left[1-\frac{c}{a}-\frac{2 \pi^{2} R^{3}}{P_{\mathrm{s}}^{2} G M}\right]\left[\frac{c}{2 a}+\left(\frac{a}{c}\right)^{2}\right]^{-1}$

and find $J_{2}=26.7 \times 10^{-3} \pm 1.9 \times 10^{-5}$. By comparison, Thomas et al. $(2005)$ have found $J_{2}=21.7 \times 10^{-3} \pm 8.5 \times 10^{-5}$. If we refer to McCord \& Sotin (2005), these two independent estimates of $J_{2}$ correspond to their internal models \#2 and \#3 for Ceres, that is to a differentiated Ceres with a silicate-rich region in its center. The rather large difference between the two $J_{2}$ determinations is due to the fact that $J_{2}$ is highly dependant on the $a / c$ ratio which shows large uncertainties depending on the measurement technique used. Lightcurve analysis for a quasi-spherical object is generally little sensitive to this parameter; while measurements made from direct imaging from the Earth distance suffer from lack of sufficient spatial resolution to estimate this parameter with the required precision. Nevertheless, both studies converge towards a differentiated asteroid and gravity field measurement made by DAWN will provide a better understanding of its mass repartition and internal structure.

Conrad et al. (2007) have shown that a detailed study of an asteroid shape, and its departure from a perfect ellipsoid, can be carried out using images deconvolved with MisTRAL. Deviations of Ceres limb measurements from our shape model can thus directly be linked to topography. Figure 3 shows that no features deviating from our shape model by more than $15-18 \mathrm{~km}$ can been observed. The highest relief expected on Ceres is calculated to be $\sim 10-20 \mathrm{~km}$ high (see also Johnson \& McGetchin 1973, Fig. 1), thus confirming that no significant deviation from an ellipsoid can be detected given the size of our resolution element. Search for relief would require a resolution of at least about $5 \mathrm{~km}$ to provide unambiguous detection of a topography. 


\section{Ceres surface composition}

\subsection{Background}

Ceres occupies a particular place in our solar system. It is physically located far enough from the Sun to have been preserved from strong heating during the T Tauri phase of the Sun (Ghosh et al. 2006, and references therein), and has possibly retained some of its primordial elements. Ceres' low amplitude lightcurve ( $\sim 0.04 \mathrm{mag}$ ) cannot result from its symmetrical shape (Thomas et al. 2005). This shows that its surface, while rather uniform, displays faint albedo features, unlike the igneous asteroid Vesta whose hemispheric albedo variations are among the strongest seen among main belt objects (Gaffey 1997). Similarly, in contrast to Vesta, whose shape is highly irregular due to past collisions (Thomas et al. 1997), Ceres displays a uniform spheroidal shape, deprived of strong surface and topographic features, and its density (see Sect. 3.3) cannot simply be attributed to macroporosity of its internal material (see Britt et al. 2002). All these characteristics point to the presence of volatile elements in the interior of Ceres. Mousis \& Alibert (2005) show that Ceres could have accreted from an assemblage of rocky and icy planetesimals, even at such short heliocentric distances. They calculated that icy planetesimals could have drifted from more distant regions of our solar system (up to $15 \mathrm{AU}$ heliocentric) to the actual position of Ceres (2.7 AU) without losing entirely their volatiles. This idea supports the model of Fanale \& Salvail (1989) in which the C/G-type objects accreted from anhydrous minerals, organics and water ice. The recent discovery of comets orbiting among the main-belt asteroids, at semi-major axes similar to Ceres' (Hsieh \& Jewitt 2006) support the possibility that small bodies can preserve part of their ices within the snow line region, which is defined as the heliocentric distance for which the temperature equals the condensation temperature of water ( $\sim 5 \mathrm{AU})$.

Other studies supporting a wet history for Ceres come from meteorites. Although no meteorites have been convincingly linked to Ceres (Feierberg et al. 1980; Jones et al. 1990; Sato et al. 1997), the C-type asteroids (whom G-type is a subclass) display a low albedo, and are thought to be the source of the Carbonaceous Chondrites (CC) meteorites. It has been shown that water ice could be stable inside CC meteorites over $4.5 \mathrm{Gyr}$ (Fanale \& Salvail 1989, and references therein), thus supporting the possibility that the Main Belt has likely experienced a hydrated stage in its history (at least its outermost part). Moreover, spectral studies of Ceres reveal a strong $3.07 \mu \mathrm{m}$ absorption band characteristic of hydrated minerals (Jones et al. 1990; Sato et al. 1997). This particular band has been the center of interest of many studies: In the early 80's, Feierberg et al. (1980) and Lebofsky et al. (1981) associated it with the signature of water frost on Ceres surface and predicted the possible existence of a polar cap. A decade later, King et al. (1992) fitted the $3 \mu \mathrm{m}$ absorption with saponite, which is an ammonium-bearing phyllosilicate whose presence in CV and CI meteorites have been suggested by Zolensky \& McSween Jr. (1988). Later, Vernazza et al. (2005) found that crystalline water ice mixed with ionirradiated asphaltite could reproduce a better fit to this feature. Recently, Rivkin et al. (2006) reviewed this past work and found that an hydrated iron-bearing phyllosilicate identified as cronstedtite, plus a few percent of carbonates could also fit adequately the near-infrared spectrum of Ceres. This recent interpretation could be supported by the detection in the mid-infrared range of emission features attributed to carbonates (from Cohen et al. 1998). In addition, iron-bearing minerals have also been invoked by Feierberg et al. (1980) and Vilas \& Gaffey (1989) to explain other spectral features present in the visible and near-infrared spectra of Ceres: $0.4 \mu \mathrm{m}$ cutoff, $0.60 \mu \mathrm{m}$ and $0.67 \mu \mathrm{m}$ weak absorption bands and $1 \mu \mathrm{m}$ shallow band (see also Vilas \& McFadden 1992). Given the radical different interpretations of the $3.07 \mu \mathrm{m}$ band reported over the past decade, it is quite impossible to conclude on its exact nature. A persistent conclusion though, consists to support the presence of hydrated minerals, or residues from aqueous alteration, in the regolith material.

As discussed above, surface water ice is not stable at distances smaller than $5 \mathrm{AU}$ and is expected to sublimate if exposed directly to solar radiation (Fanale \& Salvail 1989). Indeed, water ice migrating from the mantle region could possibly reach the surface but will escape on very short time-scale (Nazzario et al. 2003). As a result, direct detection of water ice vaporization from Ceres surface might be possible from the surroundings of a fresh impact crater, or from cracks in the sub-surface layers. Water escaping from Ceres polar region has possibly been detected in the early 90's (A'Hearn \& Feldman 1992), although this 2- $\sigma$ detection could never be unambiguously confirmed. Another supporting element comes from the relaxed shape of Ceres, which could be explained by the presence of large amount of ice in its interior (McCord \& Sotin 2005). The measurements of $J_{2}$ obtained from HST and Keck both clearly support a model of a differentiated Ceres with a volatile-rich mantle, rather than a homogeneous interior model (see Sect. 3.3).

\subsection{Near-infrared maps}

To better represent the distribution and spatial extent of the features observed on the surface of Ceres (albedo, geological marks), we projected our high-angular resolution images into maps. The following sections are used to describe in details the various steps involved in the process of map projection, and the subsequent analysis of Ceres surface properties.

Geometry: Any projection of an ellipsoidal shape onto a plane introduces deformations (Greeley \& Batson 1990). We attempted to minimize these effects by choosing the cylindrical equidistant projection, which maps the surface of the asteroid onto a cylinder tangent to its equator and conserves the distances along the meridians. As a result, this projection minimizes the deformations of Ceres' equatorial area, which is seen at highest resolution thanks to its small obliquity. Higher planetocentric latitudes suffer stronger deformations after projection, but the impact is mitigated since these regions correspond to areas imaged tangentially, at a lower equivalent spatial resolution.

Region of interest: We produced albedo maps of Ceres using the pixels located within an ellipsoidal Region Of Interest (ROI) centered on the image, and whose semi-axes were equal to $80 \%$ of the corresponding projected semi-axes on Ceres. The ROI corresponds to $64 \%$ of the projected surface for each image (see Fig. 6). Ignoring the pixels near the edge of Ceres was based on several considerations:

1- Although all images used to produce the maps were carefully cleaned before deconvolution (including from correlated noise), some of them still presented artifacts after restoration of their optimal resolution, particularly near discontinuities such as the limb and terminator.

2- It appeared difficult in a few cases to restore optimally both the contour of the object and the surface details, even if 
MistRaL is optimized to minimize the "ringing" effect introduced by the deconvolution of sharp edge objects.

3- Finally, the resolution per pixel being highest at the center of the disk of Ceres, the use of the pixels located near the edge would strongly degrade the optimal resolution of our final product.

Phase angle correction: As seen on Fig. 1, a phase angle of just a few degrees at near-infrared wavelengths produces a strong gradient of luminosity across the disk of Ceres. This effect had to be corrected prior to combine our individual images onto single maps. Several diffusion laws were investigated to model it, such as adopting a simple linear gradient, or using more complete models such as provided by the Lambert, LommelSeelinger, Minnaert and Hakpe laws (including single-scattering and multiple-scattering effects) (see Hapke 1993). The linear gradient was adopted since it provided the best fit to Ceres surface (residuals produced were nearly twice smaller than in the case of the Hapke model).

Projection: The main difficulty to project an image of Ceres into its planetocentric referential (defined by its planetocentric longitude $(\lambda)$ and latitude $(\varphi)$ as recommended by the IAU, Seidelmann et al. 2005), resides in the accurate determination of its geometrical center. As the distribution of Ceres gravity field is unknown, we made the assumption that its center of mass, which is the center of the planetocentric referential, coincides with its geometrical center. The conversion into planetocentric coordinates was based on our determination of Ceres dimensions (see Sect. 3.3) and the orientation of its spin axis (SVC, see Sect. 3.1), which is defined by the north pole angle $\left(p_{n}\right)$ and by the SubEarth Point coordinates $\left(\mathrm{SEP}_{\lambda}, \mathrm{SEP}_{\varphi}\right)$. We used our value for Ceres rotation period (see Sect. 3.2) and the Eproc ephemeris generator to obtain the $\mathrm{SEP}_{\lambda}, \mathrm{SEP}_{\varphi}$ and $p_{n}$ at the time of each observation. We then projected the images onto half-degree gridded maps to sample all areas of Ceres with sufficient resolution, from the equatorial region, up to the edge of the ROI. This fine grid map helped recover the smallest scale information from our set of 360 images.

Combination of images into maps: No absolute photometric calibration was obtained for our data. We therefore used a nearinfrared disk-integrated spectrum of Ceres (R. Binzel, personal communication) normalized to unity at $0.5 \mu \mathrm{m}$ to calculate the equivalent disk-integrated photometric value for each band, and normalize our albedo maps with respect to each other. Prior to combine the different views into maps for each wavebands, we corrected the intensity variations of the individual projections caused by the differential atmospheric absorption across our wavelength range, as well as the quality of the AO correction. We first projected each image onto the planetocentric referential of Ceres. Then we adjusted the brightness level of spatially adjacent maps by measuring the flux ratio over their overlapping area and applying the corresponding re-normalization coefficient. After correction, the maps could be combined to produce the albedo maps presented in Fig. 4 for each waveband. Each pixel of the final maps (covering a quarter of a square degree) was obtained by combining the corresponding pixels from the individual projections using a gaussian-weighted average function (we chose a gaussian with a standard deviation of 5 pixels). The largest weight (weight value $=1$ ) was attributed to the pixel providing the best spatial resolution, while the pixels with a lower resolution were assigned a lower weight. Table 6 gives the average and maximum number of images used to produce a single pixel of the final maps for each waveband, as well as the percentage of surface coverage of Ceres $(\sim 80 \%)$. In order to facilitate comparison between our near-infrared data and the UV/Visible (223, 335 and $535 \mathrm{~nm})$ HST maps, we chose to adopt the same reference meridian as Li et al. (2006a).

Maps description: The $J$-, $H$ - and $K$-band maps shown in Fig. 4 , and covering $\sim 80 \%$ of Ceres' surface (see Table 6 ), are the result of combining 126, 99 and 135 individual projections respectively. We also derived error albedo maps (Fig. 5) by measuring, for each pixel, the intensity dispersion across the individual views. The theoretical size of the resolution elements for the $J$-, $H$ - and $K$-band are $36.8 \mathrm{~km}, 47.4 \mathrm{~km}$ and $62.9 \mathrm{~km}$ respectively (corresponding to $4.4^{\circ}, 5.6^{\circ}$ and $7.5^{\circ}$ at the equator). The major features have diameters of $\sim 180 \mathrm{~km}$ (A and B) but smaller features can be seen in all three maps down to $\sim 50 \mathrm{~km}$ scale. Although the theoretical resolution is highest in $J$-band, Fig. 4 shows that the final resolution is nearly equivalent across our three bands (i.e. $\sim 60 \mathrm{~km}$ at equator). The degradation of the $H$ and $J$-band resolution is due to a more variable PSF at these wavelengths, which is supported by the larger photometric error (Fig. 5) derived for these bands. The amplitude of the albedo variation is within $\pm 6 \%$ around the mean surface value for each band. The error maps show that the albedo maps (Fig. 4) display an increasing error with decreasing wavelength: the 1- $\sigma$ uncertainty is smaller than $1 \%$ in the equatorial area in $K$ band while it is estimated to be $\sim 2 \%$ in $J$-band. The shape of the error distribution around $60^{\circ}$ longitude reveal that the linear oblique features seen in $J$ - and $H$-band are noise and should be ignored from our analysis. The $K$-band map displays the lowest noise level, due to the highest Strehl ratio delivered by the AO system at these wavelengths and should be considered as the most accurate of the three.

Some of the main albedo features in our near-infared maps can also be seen in the UV/Visible HST maps published by $\mathrm{Li}$ et al. (2006a), like the large bright area around $\left(125^{\circ},+20^{\circ}\right)$ and the dark spot at $\left(130^{\circ},-24^{\circ}\right)$. The fact that these features are visible in all wavelengths suggests that they are geological features like basins or impact craters. However the UV/Visible and near-infrared maps show also discrepancies: the bright feature at $\left(115^{\circ},-30^{\circ}\right)$ visible in the UV/Visible maps is not present in our maps, nor the dark feature located around $\left(45^{\circ},+10^{\circ}\right)$. Whereas all the discrepancies cannot be linked to surface properties (the dark annulus of the " $A$ " feature $\left(234^{\circ},+23^{\circ}\right)$ is not visible in the UV/Visible map due to its size which is comparable to the resolution element provided by the HST), such variations in their spectral behavior suggest compositional differences between these regions. The next section will provide a discussion on possible composition and geological origin.

\subsection{3-D model}

We re-projected our multicolor maps of Ceres onto its 3D shape model. Such model is used to check the distribution of albedo features present in our final maps against the features seen in our original images after deconvolution (see Fig. 6). This model can also be used to predict ${ }^{1}$ what Ceres surface would look like at any epochs, which will certainly be useful when preparing any future ground-based, or space based observations of Ceres.

\footnotetext{
1 http://www .imcce. fr/page.php?nav=en/ephemerides/ formulaire/form_ephephys.php
} 


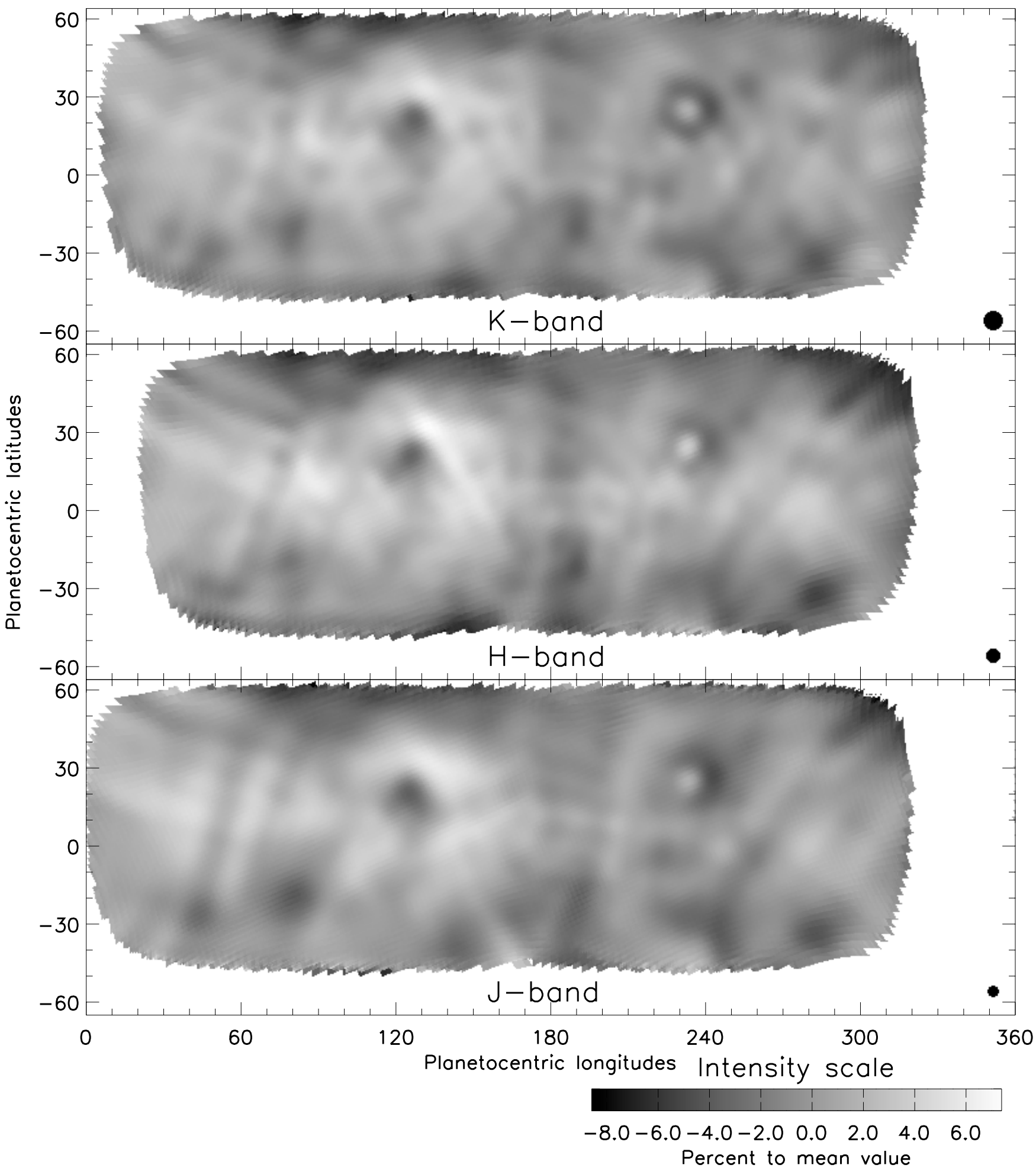

Fig. 4. $J$-, $H$ - and $K$ - band maps of Ceres covering $\sim 80 \%$ of Ceres' surface. The areas in white are terra incognita due to the limited ROI and rotational phases not imaged from Keck (see text). The theoretical resolution elements $\Theta$ at $J / H / K$ bands are shown at the bottom-right corners. Albedo variations are within $\pm 6 \%$ around the mean surface value for each map. We estimate the errors to be 2.5\% maximum (see Fig. 5). The color scale is common for the three filters. Several round shaped features are visible including a dark feature with a bright center spot at $\sim\left(234^{\circ}\right.$, $+23^{\circ}$ ) (named "A") and a dark region at $\sim\left(125^{\circ},+20^{\circ}\right)$ named "Piazzi" by Parker et al. (2002) (here "B"). Two other dark features are visible in the Southern hemisphere at $\sim\left(80^{\circ},-20^{\circ}\right)$ and $\sim\left(285^{\circ},-35^{\circ}\right)$ as well as other smaller features elsewhere (see Fig. 7). Any feature or albedo distribution present in the three maps has a very low probablity to be an artifact, with the exception of the diagonal stripes seen across the surface in $H$ - and $J$-band (see text and Fig. 5). One hemisphere $\left(0^{\circ}\right.$ to $\left.180^{\circ}\right)$ appears to be brighter $(1 \%)$ than the other at these wavelengths. A dark region running North/South is located at the boundary between the two hemispheres and is present in all maps. 
Table 6. Average and maximum number of images used to produce a single pixel of the final $J / H / K$ bands maps, and their corresponding surface coverage.

\begin{tabular}{cccc}
\hline \hline Filter & $\begin{array}{c}\text { Average images } \\
\text { (\# per pixel) }\end{array}$ & $\begin{array}{c}\text { Maximum images } \\
\text { (\# per pixel) }\end{array}$ & $\begin{array}{c}\text { Coverage } \\
\text { (\% total surface) }\end{array}$ \\
\hline$J$ & 16 & 63 & 81.8 \\
$H$ & 14 & 54 & 74.2 \\
$K$ & 19 & 72 & 80.0 \\
\hline
\end{tabular}

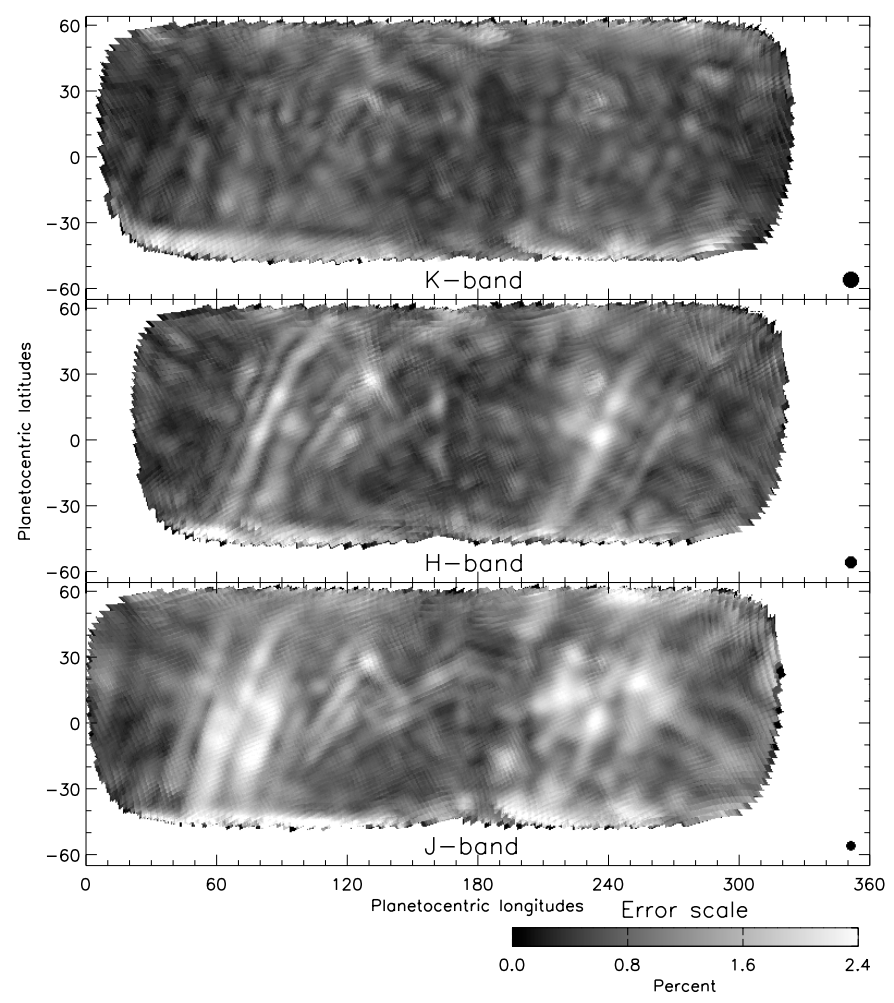

Fig. 5. 1- $\sigma$ deviation map expressed in percent with respect to the map mean value for each filter. The error is greater in $J$-band than in $K$-band as it could be expected from the lower strehl ratio at smaller wavelengths. The error also grows with latitude, which is due to the nearly equatorial view of Ceres as seen from an Earth observer.

\subsection{Three-band spectra}

We investigate the nature of the major features seen in our maps by reporting their relative brightness variation at $J-, H$ - and $K$ bands. We selected 10 type of area ( 6 bright and 4 dark, noted $b_{i}$ and $\mathrm{d}_{i}$ respectively) and show in Fig. 7 their photometric measurements normalized to Ceres integrated spectrum. The photometric value obtained for each band is the result of averaging the photometric measurements over an area equivalent to a resolution element. We estimate the error on the relative photometry as $\sqrt{\sum \sigma_{i}^{2}}$, where $\sigma_{i}$ is the standard deviation for each pixel as read in the error map (Fig. 5). The results are shown in Fig. 7 with their 3- $\sigma$ error bars.

Whereas spectral variation with the rotational phase of Ceres has never been reported, our data show differences of spectral behavior across the surface. The analysis of Fig. 7 reveals a clear trend of the bright features (left) to display a higher $H$-band albedo (with respect to $J$ - and $K$-band) than the rest of Ceres' surface. On the contrary, the dark features (right) do not display a similar trend. This may indicate a common origin for the bright features, whereas the dark regions may be the result of various surface processes, or represent different level of surface aging.

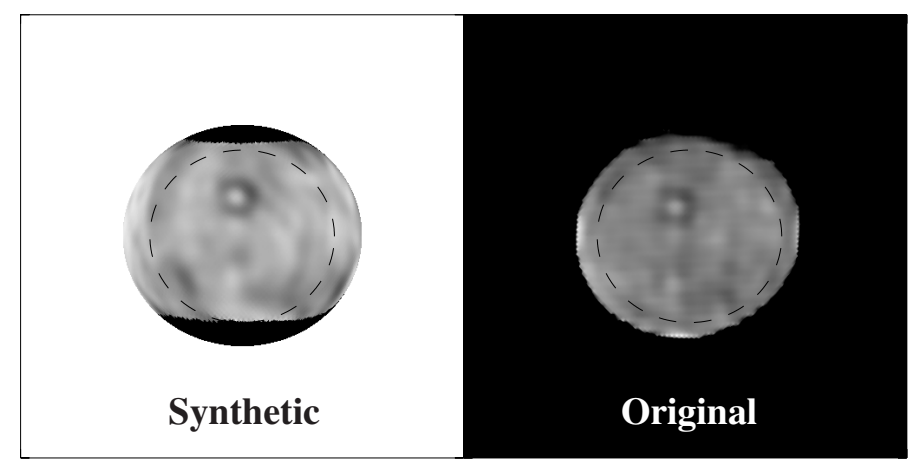

Fig. 6. Comparison between a synthetic view of Ceres (left) and a single deconvolved image (right). The overplotted dotted ellipse corresponds to the ROI limit ( $80 \%$ of projected axis). The synthetic view appears "smoother" than the original view because it is the result of the weighted average of several deconvolved images. The main albedo features are seen in both the synthetic and the original views, while other features did not resist the weighted average of several images, which acted as a filter to remove artifacts present in a single frame.

We then compare the 3-band spectra to those of various laboratory compounds. We used the ASTER ${ }^{2}$ and $\mathrm{RELAB}^{3}$ spectral libraries, to obtain the equivalent near-infrared spectra of various compounds predicted to be present on Ceres. We show in Fig. 8 their broad band photometric values, normalized to Ceres disk-integrated spectrum, each mineral being mixed with a dark neutral compound, using the mixing ratios given in Table 7 . Due to the low spectral resolution provided by our broad band imagery, it is not possible to identify unequivocally the compounds present on the surface of Ceres. We thus based our study to the comparison between the possible surface compounds proposed by Rivkin et al. (2006), who showed that carbonates like Siderite, Dolomite or Calcite mixed with phyllosilicates provide an excellent fit to the $3 \mu \mathrm{m}$ region of Ceres. Carbonates could also explain the mid-infrared spectral emission features detected by Cohen et al. (1998). We also included an orthopyroxene (Enstatite), a clinopyroxene (Augite) and Olivine, the most abundant elements in the Solar System. A water ice frost spectrum is also reported.

Although the compositional information returned at such a low spectral resolution should be considered with caution, we can use the comparison between the spectral behavior measured on the surface and that of the laboratory samples to constrain and discuss the possible surface composition of Ceres. There are similarities between the spectral behavior of the bright features seen in Fig. 7 (left) and that of phyllosilicates and carbonates in Fig. 8. The Calcite and Montmorillonite "spectra" display the same shape as the majority of the bright features. For instance, the Montmorillonite reproduces quite well the behavior of the $b_{4}$ feature as well as the bright region $b_{2}$ surrounding $\mathrm{d}_{3}$. On the other hand, the spectral behavior of the Siderite (a iron-rich Calcite), Cronstedtite (an iron-bearing phyllosilicate), or Augite (a clinopyroxene) only match the spectrum of a small percentage of Ceres surface, and for small mixing ratios show that all of them might be minor compounds of the regions discussed in this section. Igneous rocks like pyroxenes and olivine are not expected to be present on the surface of primitive

\footnotetext{
2 http://speclib.jpl.nasa.gov

3 http://www . planetary. brown.edu/relab/
} 

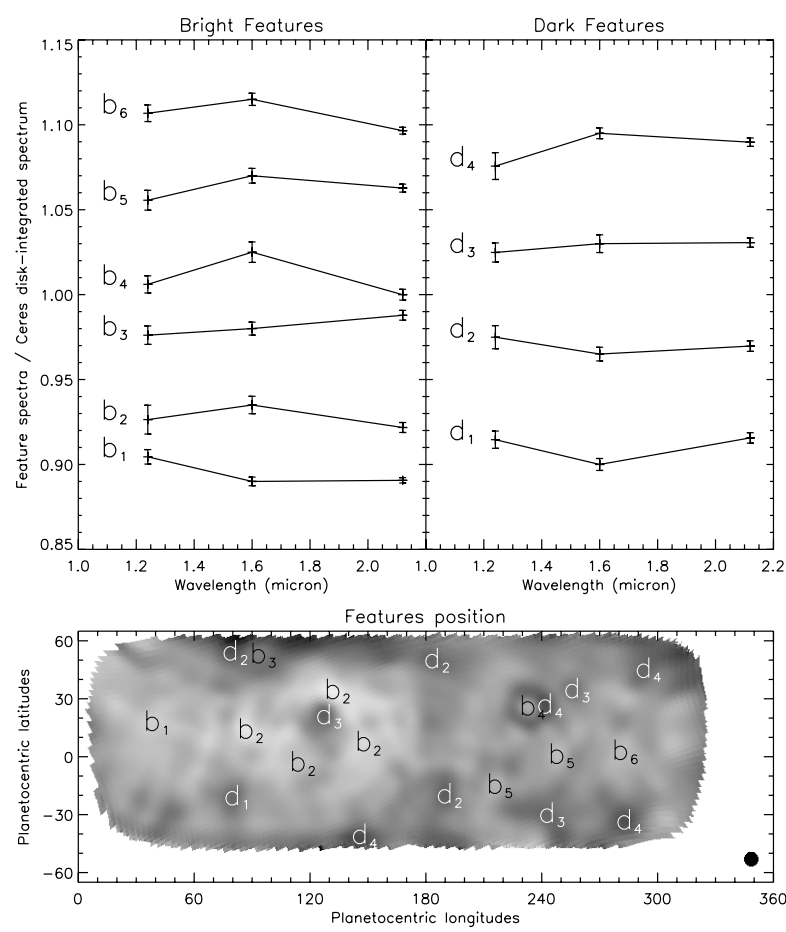

Fig. 7. Three-band spectra of selected surface features normalized to Ceres disk-integrated spectrum. The left (and right) panels show the photometric points obtained for the bright and dark features respectively. All spectra are shifted vertically by 0.045 (bright) or 0.065 (dark) to improve clarity. The letters positioned left of each spectrum are used to locate the corresponding features on the map. The $K$-band resolution element is shown at the bottom-right corner.

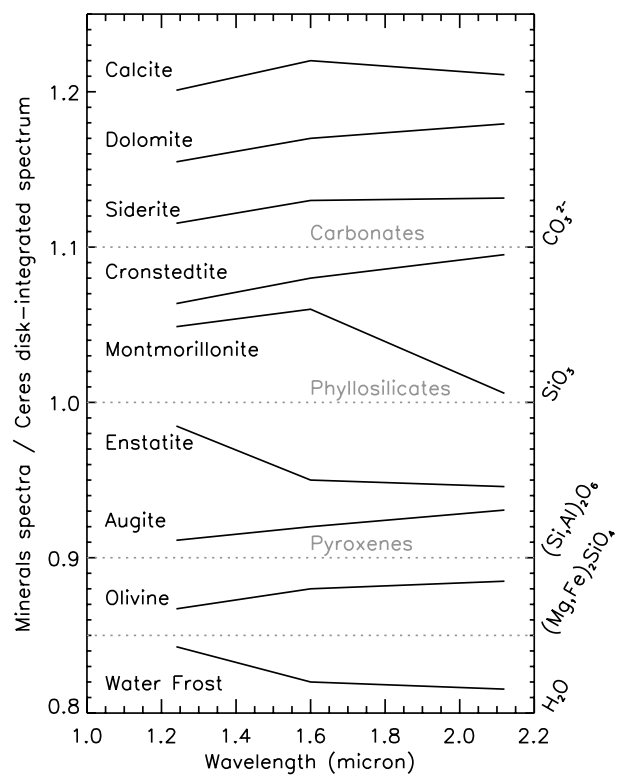

Fig. 8. Three-band spectra of selected carbonates $\left[\mathrm{CO}_{3}^{2-}\right]$, phyllosilicates $\left[\mathrm{SiO}_{3}\right]$, pyroxenes $\left[(\mathrm{Si}, \mathrm{Al})_{2} \mathrm{O}_{6}\right]$, olivine $\left[(\mathrm{Mg}, \mathrm{Fe})_{2} \mathrm{SiO}_{4}\right]$, and water ice frost $\left[\mathrm{H}_{2} \mathrm{O}\right]$ normalized to Ceres disk-integrated spectrum. Error bars are negligible.

asteroids; these compounds are likely minor components of the regions discussed in this section if present at all.

The interpretation of the dark features is more complex. Indeed, the lower $H$-band value that characterizes half of the dark features $\left(\mathrm{d}_{1}, \mathrm{~d}_{2}\right)$ does not match the behavior seen in
Table 7. Grain size and mixing ratio for the selected compounds. The last column indicates the sample reference in the spectral libraries $(a$ : ASTER and $r$ : RELAB).

\begin{tabular}{cccc}
\hline \hline Component & $\begin{array}{c}\text { Grain size } \\
(\mu \mathrm{m})\end{array}$ & $\begin{array}{c}\text { Mixing ratio } \\
(\%)\end{array}$ & $\begin{array}{c}\text { Sample } \\
\text { No. }\end{array}$ \\
\hline Calcite $^{a}$ & $0-45$ & 100 & C-3A \\
Dolomite $^{a}$ & $0-45$ & 50 & C-5A \\
Siderite $^{a}$ & $0-45$ & 4 & C-9A \\
Crondstedtite $^{r}$ & $0-45$ & 5 & CR-EAC-021 \\
Montmorillonite $^{a}$ & $0-45$ & 50 & PS-2D \\
Enstatite $^{a}$ & $0-45$ & 30 & IN-10B \\
Augite $^{a}$ & $0-45$ & 2 & IN-15A \\
Olivine $^{r}$ & $0-50$ & 5 & DD-MDD-013 \\
Water Frost $^{a}$ & $\sim 10$ & 2 & FROST.SNW \\
\hline
\end{tabular}

phyllosilicates and carbonates, which are the major compounds predicted to be present over Ceres (see Sect. 4.1). Such a drop in $H$-band matches better the behavior of Enstatite, but its high density of $\sim 3200 \mathrm{~kg} \mathrm{~m}^{-3}$ and the mid-infrared mismatch between Ceres and Enstatite spectra makes its presence unprobable. Another possible matching compound is water ice (Fig. 8). Water ice is not expected to be found in a stable form over Ceres, but "dirty" ice, i.e. ice or frost mixed with other minerals, could be present and reproduce the more elevated $K$-band point seen in the "spectra" of these features. Another point to consider is the relatively high planetocentric latitude of these dark features. None of them is found in the equatorial region, most being located above $30-40^{\circ}$ latitude. If dirty ice exists on the surface or sub-surface layers of Ceres (McCord \& Sotin 2005; Mousis \& Alibert 2005), it would be expected to be more stable at higher latitudes, where surface temperature is lower. Nevertheless, we cannot ascertain from these data alone that ice is present on Ceres. Higher spectral resolution, coupled with the high-contrast and spatial resolution provided by adaptive optics, is required to investigate in detail the composition of the main features seen on its surface. The limits of such a broad-band analysis in constraining the composition of the main features is illustrated by the bright feature $b_{1}$, which appears to match quite well the behavior expected for water frost, but it is located at low latitude and differ in reflectivity from the dark features discussed above.

The two main observed features $A\left(b_{4}, d_{4}\right)$ and $B\left(d_{3}\right.$ and the surrounding region $b_{2}$ ), were referenced as \#5 and \#2 by $\mathrm{Li}$ et al. (2006a). These two features are remarkable because whereas they sustain large physical dimensions $\left(\emptyset_{\mathrm{A}} \sim 180 \mathrm{~km}\right.$ and $\emptyset_{\mathrm{B}} \sim 350 \mathrm{~km}$ ), their spectral behavior appears to be homogeneous over such large area, which might point to a same composition and/or resurfacing history. The external annulus of the B feature $\left(b_{2}\right)$ shows the same uniformity. This annulus shape is reminiscent of a large cratering event. The bright central regions of feature A resembles the central peak seen in craters originating from high energetic impact, which would then be subject to different aging processes than the lower altitude neighboring areas. At smaller physical scale, similar differences of albedo have been reported on asteroid 25143 Itokawa between the central part and surrounding areas of small craters, this time via grain sorting (Miyamoto et al. 2007).

In summary, our AO study permits to map the albedo variations over the surface of Ceres down to a 40-60 km scale and investigate whether these variations correspond to changes in the composition. Also, the high-spatial resolution capabilities returned by AO make possible to search for areas of distinctive signature (e.g. icy rich spots), which would remain undetected otherwise in disk-averaged studies of Ceres. A dusty regolith a 
few centimeters thick (Webster et al. 1988), created by the impact of micrometeorites and possibly larger bodies, has been proposed (Witteborn et al. 1999; Lim et al. 2005) as a possible explanation for the shallow spectral signatures in the visible and near-infrared spectrum of Ceres and its small albedo constrast. But some small areas visible in our high-angular resolution images of Ceres could correspond to places on the surface where the regolith material has been cleared by "recent" endogenic or exogenic activity to expose more pristine material from the subsurface layers.

\section{References}

A’Hearn, M. F., \& Feldman, P. D. 1992, Icarus, 98, 54

Berthier, J. 1998, Notes scientifique et techniques du Bureau des longitudes, S061

Britt, D. T., Yeomans, D. K., House, K., \& Consolmagno, G. 2002, Asteroid Density, Porosity, and Structure, ed. W. F. Bottke, A. Cellino, P. Paolicchi, \& R. P. Binzel (University of Arizona Press), 485

Chamberlain, M. A., Sykes, M. V., \& Esquerdo, G. A. 2007, Icarus, 188, 451

Cohen, M., Witteborn, F. C., Roush, T., Bregman, J. D., \& Wooden, D. H. 1998, Astron. J., 115, 1671

Conrad, A., Dumas, C., Merline, W. J., et al. 2007, Icarus

Dotto, E., Müller, T. G., Barucci, M. A., et al. 2000, A\&A, 358, 1133

Drummond, J. D., Fugate, R. Q., Christou, J. C., \& Hege, E. K. 1998, Icarus, 132,80

Dumas, C., Merline, W. J., Binzel, R. P., et al. 2003, in BAAS, 956

Fanale, F. P., \& Salvail, J. R. 1989, Icarus, 82, 97

Feierberg, M. A., Lebofsky, L. A., \& Larson, H. P. 1980, Geoch. Cosmochim. Acta, 45, 971

Fusco, T. 2000, Ph.D. Thesis, Université de Nice Sophia-Antipolis

Gaffey, M. J. 1997, Icarus, 127, 130

Ghosh, A., Weidenschilling, S. J., McSween Jr., H. Y., \& Rubin, A. 2006, Asteroidal Heating and Thermal Stratification of the Asteroid Belt, Meteorites and the Early Solar System II, ed. D. S. Lauretta, \& H. Y. McSween Jr (University of Arizona Press), 555

Greeley, R., \& Batson, R. M. 1990, Planetary Mapping (Cambridge University Press)

Hapke, B. 1993, Theory of reflectance and emittance spectroscopy (Cambridge University Press)

Hsieh, H. H., \& Jewitt, D. 2006, Science, 312, 561

Johnson, P. E., Kemp, J. C., Lebofsky, M. J., \& Rieke, G. H. 1983, Icarus, 56, 381

Johnson, T. V., \& McGetchin, T. R. 1973, Icarus, 18, 612

Jones, T. D., Lebofsky, L. A., Lewis, J. S., \& Marley, M. S. 1990, Icarus, 88, 172
Keil, K. 2002, Geological History of Asteroid 4 Vesta: The Smallest Terrestrial Planet, Asteroids III, ed. W. F. Bottke, A. Cellino, P. Paolicchi, \& R. P. Binzel (University of Arizona Press), 573

King, T. V., Clark, R. N., Calvin, W. M., Sherman, D. M., \& Brown, R. H. 1992, Science, 255, 1551

Kovačević, A., \& Kuzmanoski, M. 2007, Earth Moon and Planets, 100, 117

Lebofsky, L. A., Feierberg, M. A., Tokunaga, A. T., Larson, H. P., \& Johnson, J. R. 1981, Icarus, 48, 453

Li, J.-Y., McFadden, L. A., Parker, J. W., et al. 2006a, Icarus, 182, 143

Li, J.-Y., Young, E. F., Thomas, P. C., et al. 2006b, HST Images, Albedo Maps, and Shape of 1 Ceres, eAR-A-HSTACS-5-CERESHST-V1.0., NASA Planetary Data System

Lim, L. F., McConnochie, T. H., Bell, J. F., \& Hayward, T. L. 2005, Icarus, 173, 385

McCord, T. B., \& Sotin, C. 2005, J. Geophys. Res. (Planets), 110, 5009

Michalak, G. 2000, A\&A, 360, 363

Millis, R. L., Wasserman, L. H., Franz, O. G., et al. 1987, Icarus, 72, 507

Mitchell, D. L., Ostro, S. J., Hudson, R. S., et al. 1996, Icarus, 124, 113

Miyamoto, H., Yano, H., Scheeres, D. J., et al. 2007, Science, 316, 1011

Mousis, O., \& Alibert, Y. 2005, MNRAS, 358, 188

Mugnier, L. M., Fusco, T., \& Conan, J.-M. 2004, J. Opt. Soc. Am. A, 21, 1841

Nazzario, R., Hyde, T. W., \& Barge, L. 2003, Adv. Space Res., 31, 2591

Parker, J. W., McFadden, L. A., Russell, C. T., et al. 2006, Adv. Space Res., 38, 2039

Parker, J. W., Stern, S. A., Thomas, P. C., et al. 2002, Astron. J., 123, 549

Piazzi, G. 1802, Nella Stamperia Reale, 65

Rivkin, A. S., Volquardsen, E. L., \& Clark, B. E. 2006, Icarus, 185, 563

Russell, C. T., Coradini, A., Christensen, U., et al. 2003, Plan. Space Sci., 52, 465

Saint-Pé, O., Combes, M., Rigaut, F., Tomasko, M., \& Fulchignoni, M. 1993, Icarus, 105, 263

Sato, K., Miyamoto, M., \& Zolensky, M. E. 1997, Meteorit. Plan. Sci., 32, 503 Seidelmann, P. K., Archinal, B. A., A’Hearn, M. F., et al. 2005, Celest. Mech. Dyn. Astron., 91, 203

Spencer, J. R. 1990, Icarus, 83, 27

Tedesco, E. F., Taylor, R. C., Drummond, J. D., et al. 1983, Icarus, 54, 23

Thomas, P. C., Binzel, R. P., Gaffey, M. J., et al. 1997, Science, 277, 1492

Thomas, P. C., Parker, J. W., McFadden, L. A., et al. 2005, Nature, 437, 224

van Dam, M. A., Le Mignant, D., \& Macintosh, B. 2004, Appl. Opt., 43, 5458

Vernazza, P., Mothé-Diniz, T., Barucci, M. A., et al. 2005, A\&A, 436, 1113

Viateau, B., \& Rapaport, M. 1998, A\&A, 334, 729

Vilas, F., \& Gaffey, M. J. 1989, Science, 246, 790

Vilas, F., \& McFadden, L. A. 1992, Icarus, 100, 85

Webster, W. J., Johnston, K. J., Hobbs, R. W., et al. 1988, AJ, 95, 1263

Witteborn, F. C., Cohen, M., Bregman, J. D., et al. 1999, AJ, 117, 2552

Zolensky, M., \& McSween Jr., H. Y. 1988, Aqueous Alteration, Meteorites and the Early Solar System ed. J. F. Kerridge, \& M. S. Matthews (University of Arizona Press), 114 